\title{
Producer Mobility Support Schemes for Named Data Networking: A Survey
}

\author{
Muktar Hussaini, Shahrudin Awang Nor, Amran Ahmad \\ InterNetWorks Research Laboratory, School of Computing, Universiti Utara Malaysia, Malaysia
}

\begin{tabular}{l}
\hline \hline Article Info \\
\hline Article history: \\
Received Feb 14, 2018 \\
Revised Jun 29, 2018 \\
Accepted Sep 16, 2018 \\
\hline
\end{tabular}

\section{Keyword:}

Information centric networking

Mobility approach

Mobility support scheme

Named data networking

Producer mobility

\begin{abstract}
Mobile devices connectivity and data traffic growth requires scalable and efficient means of data distribution over the Internet. Thus, influenced the needs for upgrading or replacing the current Internet architecture to cater the situation as Named Data Networking (NDN) was proposed. NDN is cleanslate Internet architecture, proposed to replace IP with hierarchical named content that utilizes route aggregation to improve scalability and support mobility. Although, NDN provides supports for content consumer mobility with the help of catching capabilities, however, content producer faces many problems similar to mobility in IP architecture, such as, long handoff delay, unnecessary Interest packet losses and high bandwidth utilization. Hence, many concepts and schemes were proposed to address these problems. This paper reviewed and conceptually analyzed the schemes based on their fundamental design that broadly categorized into indirection-based approach, mapping-based approach, locator-based approach and control/data planebased approach. In the review analysis, mapping-based approach schemes provide optimal path for packets delivery, high handoff delay Indirectionbased and locator-based approach schemes provide normal handoff delay, but introduces tiangular routing path. The control/data plane-based approach schemes provide sub-optimal routing path and high handoff delay. The paper provided both strength and weakness of each scheme for further research.
\end{abstract}

Copyright $@ 2018$ Institute of Advanced Engineering and Science. All rights reserved.

\section{Corresponding Author:}

Muktar Hussaini,

School of Computing,

Universiti Utara Malaysia,

06010 UUM Sintok Kedah Darul Aman, Malaysia.

Email: elhussenkaz@yahoo.com

\section{INTRODUCTION}

Named Data Networking (NDN) architecture is a clean-slate architecture that achieved data authenticity, confidentiality and integrity, to replace the thin waist of networking stack of IP architecture with named content. Naming becomes the most significant part of application design of NDN that qualifies support for multicast, content distribution, delay-tolerant networking and mobility [1]. By default, Information Centric Networking (ICN) proposed to support mobility and other IP shortcomings, but many challenges aroused especially in NDN with regards to mobility support. Mobility support allows mobile devices to relocate between different access point without disrupting the content availability and minimal hand-off delay. Hence, the mobility in ICN was divided into consumer, producer and network mobility. Producer mobility is the support for the content producer to relocate without disrupting consumers and intermediate routers for content availability and its location within minimal hand-off time [2]. However, many researchers acertain the unsopprt for producer mobility in named data networking architecture.

Zhu, Afanasyev and Zhang [3] ascertain the unsupport of producer mobility in NDN. Even though, caching improves the performance and smoothens the mobile's handoff for consumer but it faces similar problems for mobile producer as in IP architecture, because of routing table size scaling problem still exist, 
especially in dense area with high mobility [4]. To provide solution to the problem, Zhu et al. suggest separation and mapping between identifier and locator by broadcasting or intermediate nodes such as DNS servers [3]. Also, NDN uses hierarchical naming and route aggregation to improve scalability, but naming structure generates substantial challenges when providers move to different location and introduce significant scalability challenges. Hence, the problem can be lessened via caching, replication and provision of highspeed mobile producer hand-offs [5]. Ahlgren et al. [6] emphasizes the natural support of content consumer mobility in NDN, but problems may arise for content producer mobility. Also, expressed that, in consumer mobility there is no need to keep an association to a specific copy alive, instead, new connections can be establish and alternative copies cached at the new location can be used [6]. NDN use Named-Based Routing (NBR) and hierarchical naming to aggregate route announcements. For a moving object new route needs to be announced and propagated to replace old routing information causing similar problem as in IP. Besides, producer mobility in NDN caused a serious problem that offers long handoff latency and unnecessary Interest packet losses on transmission towards the old location of the producer. In addition, causes congestion that affect the integrity of data [7].

Recently in survey articles for named data networking and mobility support in NDN by [2] and [8] reported that consumer mobility was inherently supported in NDN while Producer mobility cannot be support and faces many challenges from initial architectural design of NDN. Nevertheless, there are different proposal to solve the producer mobility problem such as mapping based and locator free techniques [8]. In addition, Feng et al. [2] expresses that, although consumer mobility formerly supported in NDN by means of network caching, but still there are some problems need to be addressed and yet producer mobility was not supported as a result of content named are not separated with location. In addition, another means is required to serve as a locator [2] to support producer mobility in NDN.

This paper is the review and analyses mobility support schemes for content producers in NDN; and provides schemes classification for producer mobility support approaches based on their fundamental design, specifically for NDN. The rest of this paper is arranged as follows: Subsequently for the general introduction, in section two the main concepts of producer mobility support for NDN was described. Section two analyses the strengths and weaknesses of four different approaches of producer mobility support schemes, reviewed and analyzed based on individual scheme characteristics. Section four is the review analysis for different approachs and thier research trend based on years and finally the conclusion section.

Many researches have been carried out an attempt to provide solution to producer mobility in NDN; some proposed certain concepts and schemes as solutions. Zhu et al. [3] presented three different conceptual schemes as a new perspective to support mobility in NDN. The concepts established the fundamental procedure to solve producer mobility problem for other researchers, also the concepts were recommended to support mobile producer from the lesson learned in previous researches that provides IP mobility support solution which can be applied directly to solve producer mobility support in NDN. The three conceptual schemes are, using mobile producer storage to fetch data in the absence of producer, using intermediate nodes such as Domain Name System (DNS) servers, anchor, rendezvous and Interest broadcasting for mapping between content identifier and location [3]. Broadcasting is a well known techniques used for different mobility and protocols solution in computing [9], broadcast scheme was proposed by [10], [11]

Hermans, Ngai, and Gunningberg [12] ascertain that to provide a concrete solution for producer mobility, content identifier and content locator decoupling is necessary. Added that, many challenges were raised to solve the mobility support problems such as, preferable namespace to be used for content identifier and content locator, provision of session continuity, how to resolve identifiers and locators using mobility anchor or explicit identifier [12]. In order to retain IP routing principles, the locators must be in hierarchical form of namespace and the node can be able to handle the mobility, hence separation of identifiers and location together with explicit identifier resolution is required to support producer mobility and provide the scalability of routing infrastructures [12]. Therefore, the existing proposed schemes and classification approaches were initiated from these conceptual solutions provided.

\section{PROPOSED SOLUTIONS OF NDN PRODUCER MOBILITY SUPPORT}

Some researchers classified existing producer mobility support approaches into different classes for different application purposes and easy identification of their strengths and weaknesses. Producer mobility support approaches were identified as rendezvous point based or home repository and indirection point based [13], [14], to provides seameless mobility as improved in MIPv6 for handover enhancement [15] and multicast management mobility in IP [16]. Similarly, Saxena et al. [8] further classified producer mobility support approaches as mapping-based approach and locator-free approach. Also, mobility support approach in NDN for both consumer and producer were classified as partial separation and temporary separation by [2]. Therefore, in this paper the main concepts schemes were broadly divided into Indirection-Based Mobility 
Approach (IBMA), Mapping-Based Mobility Approach (MBMA), Locator-Based Mobility Approach (LBMA) and Control/Data Plane-Based Mobility Approach (CDPBMA) according to the conceptual solution presented in previous section. Many solutions were provided based on these approaches

\subsection{Mapping Based Approach}

The Mapping-Based Mobility Approach (MBMA) is a technique adopted by many researchers [3], [17]-[21] to provide producer mobility support schemes that depend on a DNS server, rendezvous point and mapping server that keeps mapping track of content identifier and current location for mobile producer. Once the mobile content producer move and connect to the new Point of attachment (PoA), the producer discovered a new name prefix and send signaling update to the DNS [17], [18], rendezvous or mapping server [20], [21]. The DNS server processes all necessary mappings between the content identifier or new domain name prefix and its location. The consumer on the other side, send a query to the mapping server seeking the new location of content provider that changed location. Then, the mapping server reply the query with forwarding hint as new location update, which contains the mapping of domain name prefix and its location of the content Producer. The consumer uses the forwarding hint and forwards the Interest to mobile Producer at its new location, as shown in Figure 1 (a).

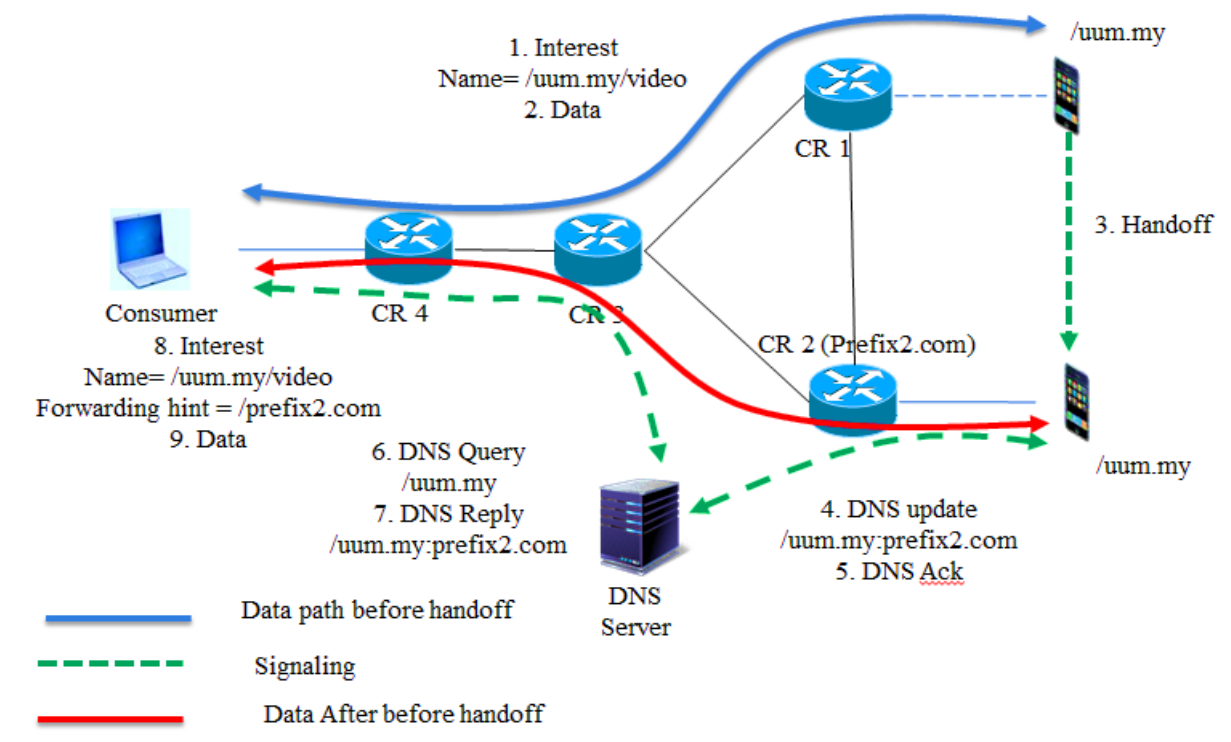

Figure 1 (a) Operational Model of MBMA
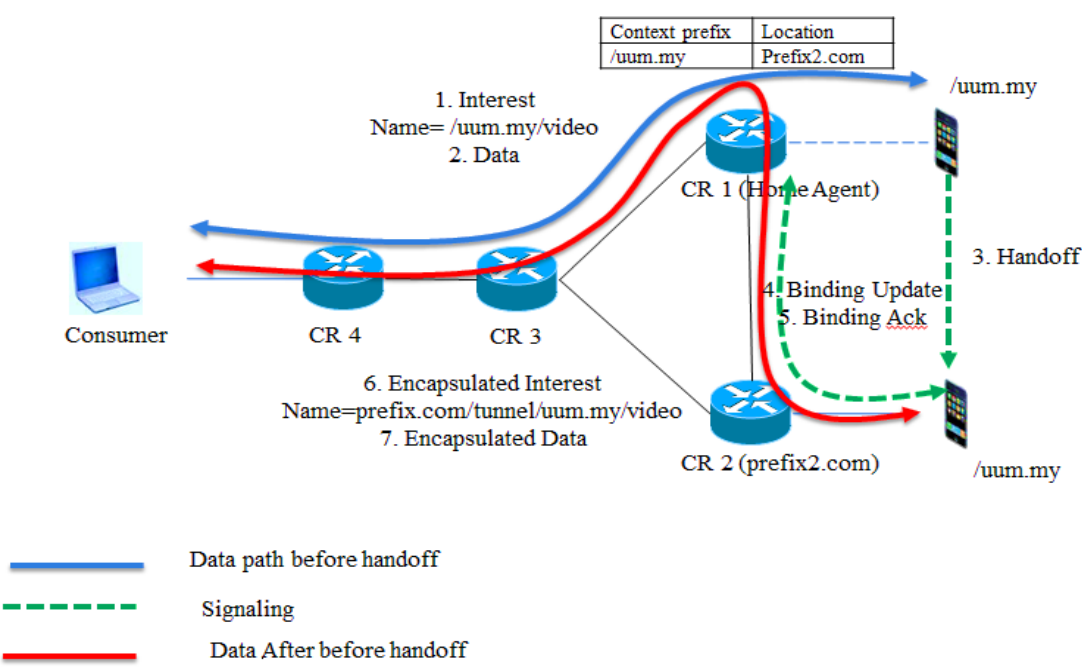

Figure 1 (b) Operational Model of IBMA 
The coupling or mapping between the identifier name and the locator name can be provided by using either a broadcasting or intermediate node [3]. A namespace can be provided for each mobile producer under which its publish its content regardless of the location to serves as identifier of the mobile producer, and another name prefix for the access router should be provided to serves as locator that can be used to locate the new PoA of the mobile producer [3]. If any consumer wants to access data from producer that relocate to new PoA, the consumer may send a request to the intermediate node (DNS server) for updated information of the content producer, the information can be used as forwarding hint.

Kim et al., [17], [18] proposed three mobility support schemes to curtail the impact of handoff delay while providing service especially on delay constraint data, among the schemes the rendezvous server was used to keep the track new location of mobile Producer when there is occurrence of handoff and update the clients or content consumers after mapping between content identifier and new location of the producer. However, the maximum handoff latency of rendezvous scheme is around 400ms [17], [18], which is far greater that the Round Trip Time (RTT), hence the rendezvous server can serve the consumer with outdated information.

Jiang et al., [20], [22] provides a solution to the content producer mobility in NDN using DNS-like mapping approach and adopt forwarding hint to explore the benefit of NDN in supporting mobility. The mapping of content identifier and new location of the mobile producer was carried out by the DNS server and the forwarding hint is added in to the Interest upon request by the consumer [20]. However, in this solution each application domain required to have independent mapping server and implemented as distributed systems. Also, mobile producer must update its new location to the server for the mobility to sustained, causing high network signaling and overhead.

Another mapping-based approach mobility support scheme was proposed by Gao and Zhang [21] to solve the producer mobility problem in NDN without tampering the original NDN architecture. The scalable mobility management (SMM) scheme adopts three different separation mechanisms, access/core separation, locator/ID separation and management/routing separation. The scheme was proposed to address some problems such as severe scalability, non-optimal routing, single point of failure and the result outperforms mobile IP schemes in terms of communication and handoff latency [21]. However, to prevent single point-offailure distributed mapping system was provided in SMM, which can result to the extra delay and overhead cost for the management of global binding update. In addition, to improve and minimized delay during handoff process, fast handoff as in [23] was proposed to use tunneling of Interest packets and binding update, as well as data packets and binding acknowledgement, which may result of worsen bandwidth utilization, especially when there is frequent movement of mobile producers.

Table 1 summarize some schemes under the approach of mapping-based, the method of mobility information update was highlighted, together with strength and weaknesses of each scheme. In general, mapping-based approach provides optimal routing path for data packets delivery after handoff. However, the approach has high signaling cost and handoff latency, with partial modification of NDN architecture.

Table 1. Mapping-Based Mobility Approach Schemes

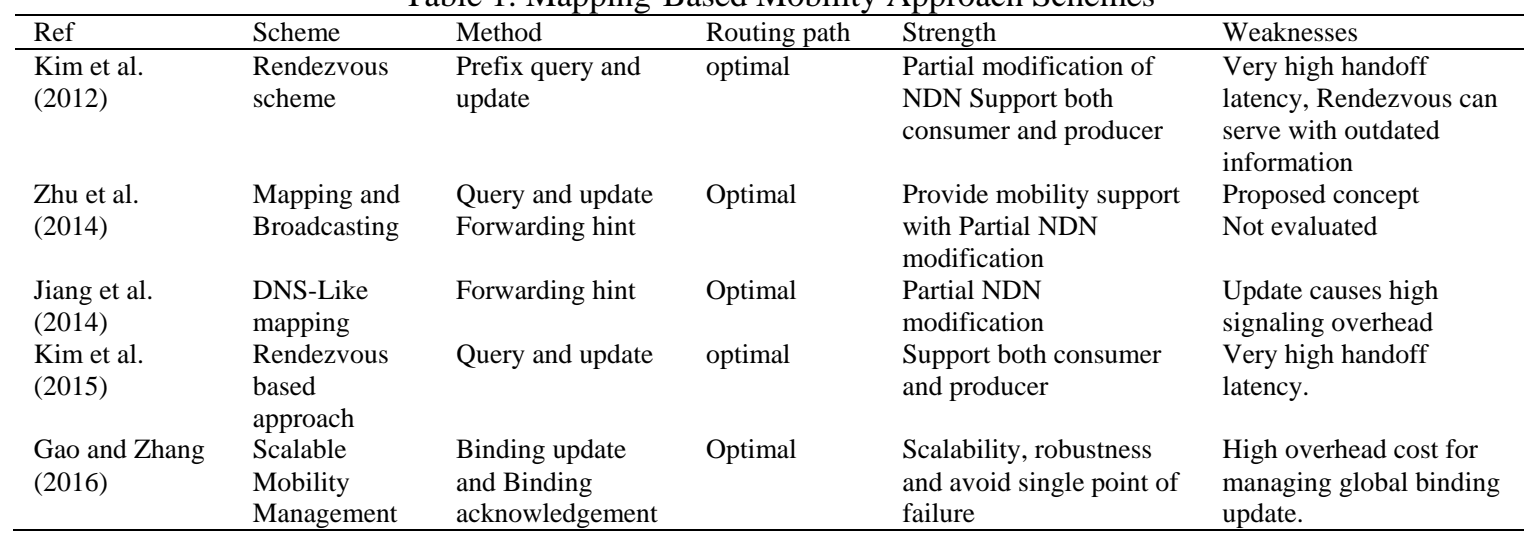

\subsection{Indirection Based Approach}

The Indirection-based Mobility Approach (IBMA) is a technique derived from the MIPv4 and MIPv6 mobility solution concept to support the mobile producer in NDN architecture [8]. A home agent or home router was provided to maintain the binding information between content prefix and location and redirect the Interest packets using tunnel to the new location or PoA of the mobile producer [24], [25]. The producer or content provider registers its content prefixes with the home router as in [25], or Indirection point 
as in [24] that directs all the Interest packets from the consumer to the producer and reverse the data packets in breadcrumb nature of NDN. When the producer decides to move, after the handoff from the current location, it sends binding information as an update notifying the home router about the new location. Once the home router receives the subsequent Interest packets from the same routing path, the Interest are being encapsulated and tunneled directly to the new provider's location or PoA, as illustrated in Figure 1 (b).

Hermans et al. [24] proposed an Indirection point scheme to support Producer mobility in NDN by adopting the concept of mobile IP, but the solution handles Producer mobility seamlessly without extensions as in Mobile IP. The scheme utilized target prefix identifies the context name and source prefix to identify new location or PoA of mobile producer in another network domain. The mobile producer must register its target prefix first, for the Indirection point to announce it to the network, also updates its source prefix to the Indirection point that keep the binding information of target and source prefix. For any change of location, the mobile producer sends a new binding request to the home repository or indirection point to update and delete the old binding information. When indirection point receives an Interest requesting a particular target prefix and the content not available in the cache, it performs longest prefix matching of Interest in the binding table, once found the Indirection point encapsulate the original Interest in a new Interest and forward it to the mobile producer. When producer received the Interest, it de-capsulate the Interest and tunnel back the data packet to the Indirection point. The indirection point de-capsulate the data and send it to the consumer [24].

Lee et al. [25] also proposed an indirection approach similar to [24] that uses home domain Content Router $\left(\mathrm{CR}_{\mathrm{h}}\right)$ and intermediate Content Routers (CR) to handle Producer mobility. The mobile producer always sends a prefix update to $\mathrm{CR}_{\mathrm{h}}$ about its movement to new location; also announce the prefix to the new $\mathrm{CR}$. The new $\mathrm{CR}$ forward the prefix update to the $\mathrm{CR}_{\mathrm{h}}$ to establish path from the new and old domain of mobile producer. Afterward the Interest and Data packets are exchanged between mobile producer and consumer through $\mathrm{CR}_{\mathrm{h}}$ by tunneling encapsulated and decapsulated Interest and data package as in [24]. However, for the intermediate content routers to cache the content, they must have to decapsulate the content from tunnel header and cache the content with its original name prefix. In addition, the nature of Interest and data packets transmission through the home content router may result in a problem of one single point of failure of $\mathrm{CR}_{\mathrm{h}}$ and tunneling-base redirection provides problems of triangular routing as in mobile IP.

In addition to rendezvous scheme mobility support, Kim et al. [17], [18] proposed a scheme using Indirection point to support producer mobility by reducing the routing update overhead. The scheme was introduced to minimized the handoff and improve the performance of Rendezvous server scheme that induces large handoff latency. Indirection point serves as a relay point between content Consumer and mobile content producer [18]. The scheme reduced the latency upon rendezvous server scheme to maximum handoff latency of 226ms [17] and approximately 200ms [18] this indicates that the performance of Indirection server mobility support is more efficient than Rendezvous server. However, despite the less handoff latency than rendezvous, the RTT is longer almost twice that of rendezvous, and all the data are exchanged through the indirection server consequently becomes a single point of failure.

The improvement of handoff performance can also be degraded as a result of longer RTT for the Indirection point scheme. Kim et al. [17], [18] proposed Interest forwarding scheme without changing of new hierarchical name when content producer is in mobile. The changing processes demand a lot including mapping and registration to rendezvous server. The Interest forwarding scheme processes starts when the content Producer notify its access router about the handoff process, the access router buffers any interests heading to mobile Producer after the notification. After the handoff, the new access router forwards a virtual Interest to the old access router by longest prefix matching in FIB for intermediate router to update their FIB, once the virtual Interest reach old access router its forward the buffered Interest to the new access router without forwarding hint. The data requested are delivered directly to the consumer as the PIT in one of the intermediate router direct it to the consumer, thus the routing path to mobile producer is successfully updated, the subsequent Interest is forwarded directly to the new location [17], [18]. In comparison with other approaches proposed, the handoff latency are minimum 178ms [17] and 160ms [18] and RTT is 54.43ms [17] are also minimum compared to rendezvous and Indirection scheme. Besides the minimizations of handoff latency, the Interest forwarding scheme has advantages of localizing path update between old and new access router to avoid the explosion of routing table size. However, the scheme requires the modification of routers to buffer incoming Interest during handoff and processes of virtual Interest. In addition, the localize path update may result in problem of triangular routing in some topologies.

Han et al. [26], [27] proposed a publisher mobility support that is partially similar to an indirectionbased scheme that uses two reserved names to manage and control the mobility of content producer. In [27], the conceptual idea was proposed without details design and evaluation on how to support mobile producer by providing fast FIB establishment and maintaining valid path to reach the content producer after the handoff between old and new PoA. In [26], the proposed protocol was design, evaluated and benchmark 
against purely indirection approach and traditional CCN architecture. The names were used to update the home router and old PoA about the mobility event of content producer [26], [27]. For the normal process when the producer does not move, all interest packets were forwarded through the home router using static FIB. Moreover, to maintain the mobility, one name is used to update the home router and other is used to update the previous or old PoA with path information to the producer, using dynamic FIB. However, the two names were added to the data and interest packet names, which may result to the high signaling overhead cost and bandwidth exploitation when there is frequent movement of high number of mobile producers. Although the solution provides sub-optimal path for data transfer between consumer and producer, still the interest packets must be forwarded to the home router and subsequent old PoAs before transferring it to the content producer which can lead to the high delay of content retrieval.

Yan et al. [28] proposed a distributed mobility management scheme as a solution for both consumer and producer mobility in NDN architecture and all mobile IP architecture supported by NDN overlay [19]. The distributed mobility management handover scheme, supports both consumer and producer mobility which minimized handoff latency and routing update cost or signaling overhead cost. In addition, when mobile produce moves from old to new PoA, the proposed approach selected a branching node of old and new PoA to dynamically adjust the routing update accordingly. Thus, signaling cost reduced significantly due to the hop-by-hop state update after selection of branching node. However, in this solution the Interest packets received by the home domain router must be encapsulated and forward it to the foreign domain router, to the mobile. Similarly, the data packet must be encapsulated and forward to the home domain router, de-capsulate and forward to the consumer. Therefore, the process of encapsulation and de-capsulation increases the overhead cost of the network, especially if the number of mobile producers increases significantly.

\subsection{Locator-Based Approach}

The Locator-based Mobility Approach (LBMA) is a technique based on separation of content identifier and locator, the assigns a unique locator field for access router or home router that maintains the processes of mapping between the Identifier or content prefix of the mobile producer and locator of its new location [12]-[14]. Figure 2 (a) illustrates the operation of the LBMA, when the mobile producer decided to move to another location, in the processes of handoff by the producer, it sends an update of its new location to the home router. The consumer still sends the Interest packets to the normal route reaching the home router, the home router modified the Interest packets and adds the current location of the mobile producer, and then routed the modified Interest packets based on the location information added towards the new PoA of the producer.
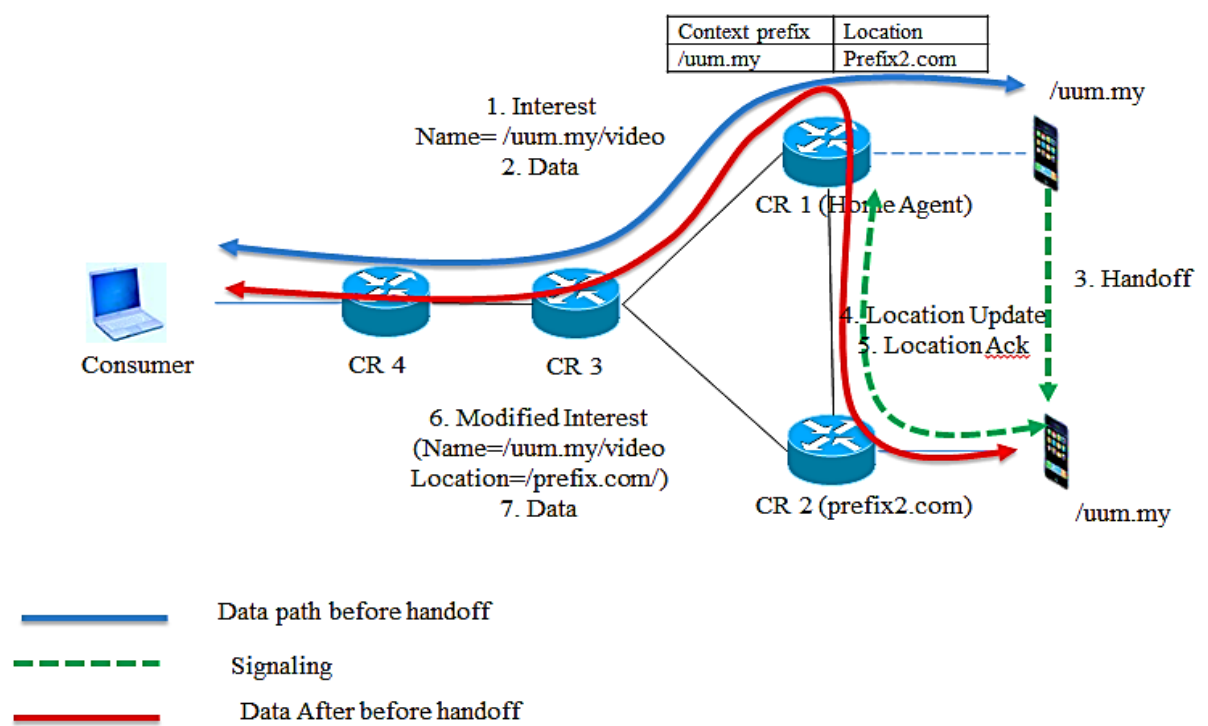

Figure 2 (a) Operational Model of LBMA 


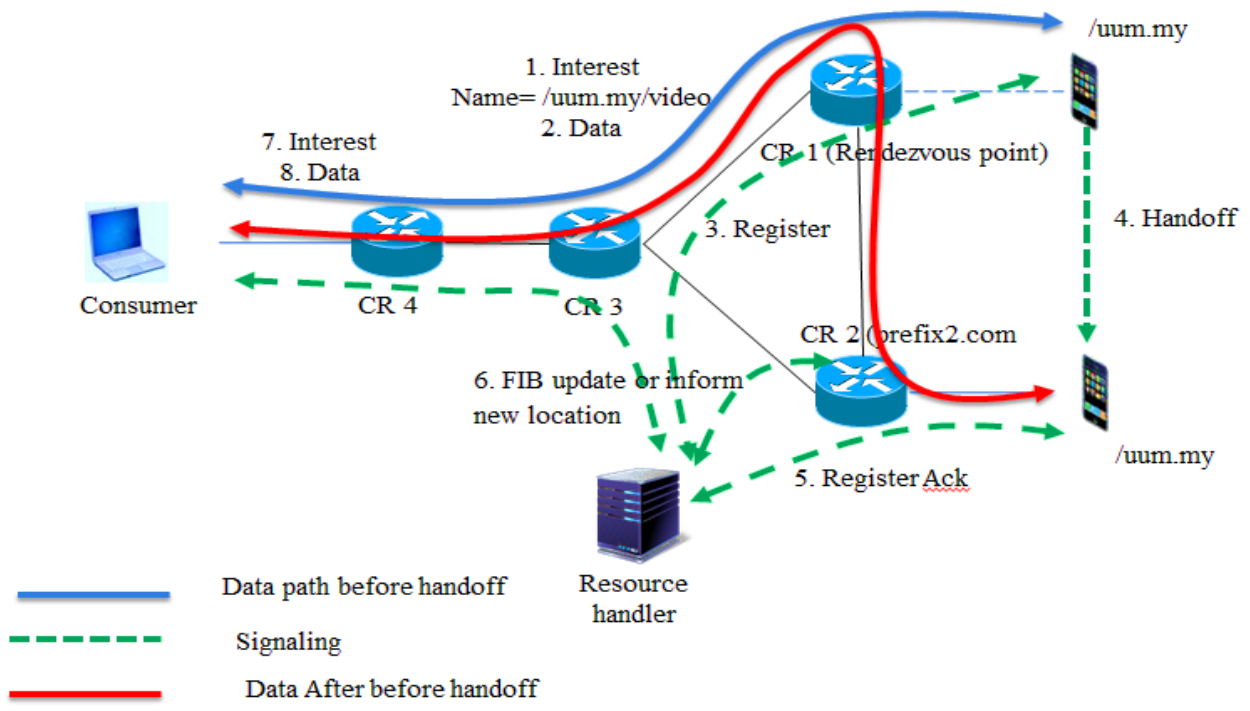

Figure 2 (b) Operational Model of CDBMA

Rao, Luo et al. [13] and Rao Gao et al. [14] proposed Locator-Based producer mobility scheme by adding a locator to each AR (Access Router) in NDN network as unique topological layer, also extend the functionality of AR with capability of catching, forwarding Interest packets on behalf of the source and added a field in the original NDN packet. FIB entries that contains only list of interfaces is modified to include list of mobility status and locator. The scheme was called locator/identifier separation-based approach [23] where the mapping of producer's content prefix that serves as identifier and its current location take place by home router. However, the solution provided better handoff performance over RBMA and slight improvement upon IBMA, but when numbers of mobile nodes were increases the performance will be degraded below IBMA. In addition, the solution cannot be suitable for a very large network when the cost between new and old access router increases.

Hermans et al. [12] proposed location-based mobility scheme similar to Mobile IP, but uses mobility anchor without encapsulation. The normal format of interest packet is modified to accommodate an optional field for location name of the mobile content producer in addition to the normal content name. After the handoff when a router receives normal Interest the router propagates it on the content name as normal, but when the received Interest contain location name field and the router do not have the requested content cached it will propagate the Interest to the content producer. In that situation at each caching router the location name requires to perform two lookups to match the content name and location name to determine the next hop that needs to forward the Interest [12]. However, the packets delivery cost become higher when number of mobile producers increases due to the double lookup to determine the next hop. In addition, the handoff latency can affect the overall handoff performance.

Location-aware on-demand multipath protocol based on NDN MANETs was proposed by Asif and Kim [29] to solve the intermittent connectivity, low battery power, data redundancy, packet flooding that affect network performance due to the nature of broadcast nature of wireless mobile ad-hoc network in NDN. A relay node was created to utilized multipath forwarding of data and Interest packets to reduce unnecessary flooding of data or interest packets, for perfect utilization of broadcast nature of relay node. The Interest packets or data follow multiple routes, if the producer's location is not known, the relay will rebroadcast the Interest packets until producer is located [29]. The performance of proposed scheme was benchmark against provider-aware forwarding and blind forwarding [30] that used broadcasting transmission mechanism, the result shows that location-aware on-demand multipath have better content retrieval and Interest retransmission. However, the solution cannot be suitable for general NDN architecture as its targeted NDN MANET only.

In the study of Azgin, Ravindran and Wang [31] anchor chain on-demand mobility support solution for ICN was proposed to manage producer mobility and handoff processes to avoid data loses. The architecture used decentralized micro-level resolution system and chained distributed anchors that provide forwarding functionalities to help for the efficient packets forwarding to the mobile producer after handoff [31]. The architecture performed better in respect of forwarding efficiency and handoff latency [31]. 
However, the solution imposed high signaling as a result of pre-handoff, post-handoff messages from the mobile producer and path remove messages when anchor removed from the path.

Table 2 summarize some schemes under the approach of location-based, the method of mobility information update was highlighted, together with strength and weaknesses of each scheme. In general, LBMA provides normal handoff latency, signaling cost and partial modification of NDN architecture. However, the approach did not consider path optimization after handoff and the central node (Home repository, Anchor, Access router) utilization can be resulted in path stretching.

Table 2. Location-Based Approach Schemes

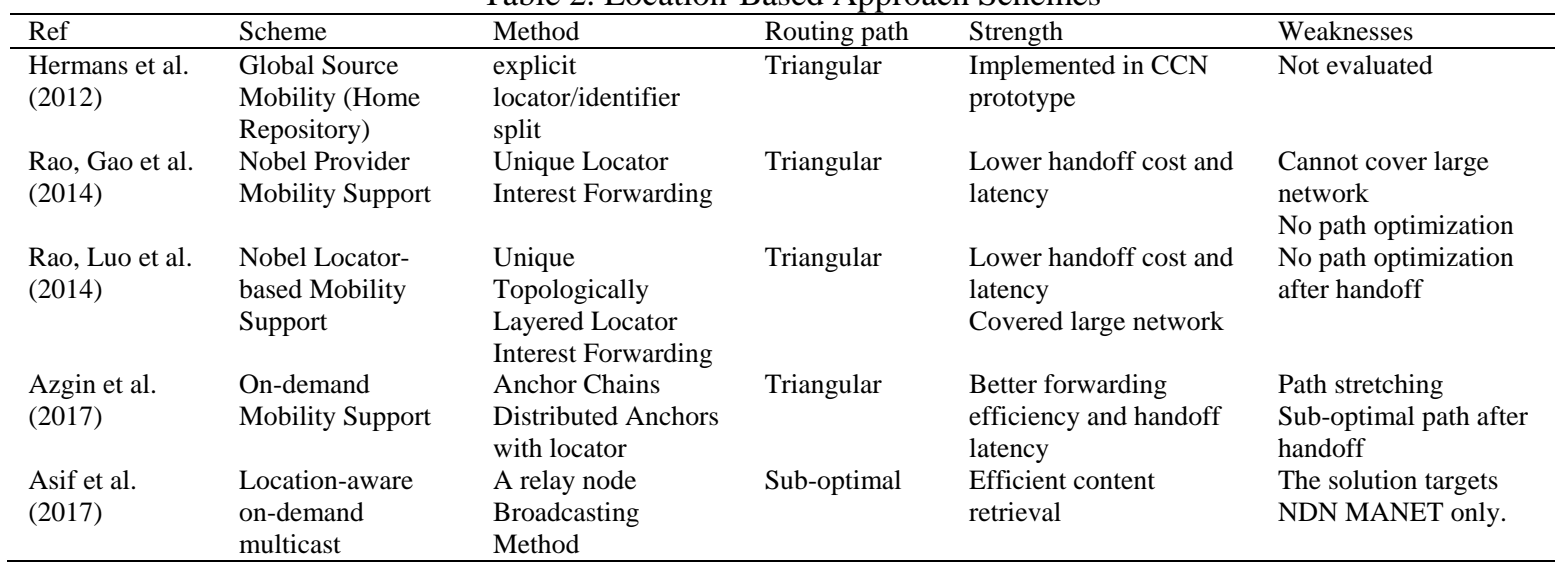

\subsection{Control/Data Plane- Based Mobility Approach}

The Control/Data Plane-based Mobility Approach (CDBMA) is a technique based on control plane and data plane separation to support mobile producer. The control plane used a server that serves as controller [32] or resource handler [33] to control the process of mobility signaling during the handoff. The data plane on the other hand is responsible for the forwarding of Interest packets and Data packets after the handoff. Figure 2 (b) shows the mode of operation of CDBMA for producer mobility support. During the handoff, the producer registers its new location to the controller or resource handler. The control plane updates the data plane about the new location of mobile producer.

Tang et al. [33] used the idea of control and data plane separation together with locator and identifier separation to provide producer mobility scheme solution for both intra and inter-domain mobility of content producer with minimum handoff latency. The locators' names were categorized into global and local locator which is added in an optional field in the Interest as a hint to assist the routing of Interest. Global locator is used to locate Rendezvous Domain (RD) where content producer located and the local locator is used to locate the content router which the mobile content producer attached in the domain.

In addition, a locator update Interest packets was provided to contain the biding information for the content name and new location of mobile producer. The Resource Handler (RH) was provided in each RD and a Rendezvous Point (RP), the RH notifies the RP about the new location and a global locator when content producer attached to the new location (content router). The RH manage the registration and signaling update for any location changes in control plane and the Interest and data packets are routed through RP [33]. However, the handoff processes of the scheme were analyzed for both inter and intra-domain Producer mobility, where the result shows lower handoff for intra-domain and very high handoff for inter-domain movement.

Unlike in [33], Zhou et al. [32] uses controller to provide central control system knowing the topology to minimize Interest and Data losses, handoff delay, when mobile producer change to new location. Each Autonomous System (AS) keeps and maintained one controller that can acquire the information of the links and topology of the region and can communicate with each other to exchange control information using control message packets to support mobility handoff. The two separate planes were control plane and forwarding plane, where control plane take the responsibility of control information management and exchange of control message, while forwarding plane is in charge of Interest and Data package forwarding [32].

The scheme operated in some stages after the network initialization for obtaining the network topology information. The first stage is for the content provider to register its new content through the attached content router to the controller that adds the contents records and broadcast the content into the network. When content producer relocates, there is a need to delete the record and update with new location 
information. The controllers update the FIB table in the intermediate nodes or content router for directing the Interest packets to the mobile content producer. However, the handoff processes accumulate high signaling cost and the solution is more of software defines network architecture which is not compliance with NDN architecture.

To reduce the handoff latency and minimized the signaling overhead Ren et al. [34] proposed a producer mobility management scheme using Software Define Controller (SDC) to improve the performance of [33] scheme. The basic idea of the proposed scheme is to separate control from data plane as in [32], [33], updating routing tables entries and configures the relevant content routers by the SDC. In this scheme [34], a routing table that will updates, rewrites and send the configuration of route entries was designed. In addition, when mobile producer moved to another location there is no need for change of the original content name, and the packets (data and Interest) are forwarded according to the new update provided by SDC.

Greedy routing was implemented to support both consumer and producer mobility in receiver driven data-centric paradigm called MobiCCN, which can be applied to any ICN architecture such as NDN [35]. The scheme was characterized by nearly similar set-up of control/data plane-based approach, but not exactly as it is uses two different routing protocols for static and mobility situation. The protocols are greedy routing protocol coexist with $\mathrm{CCN}$ routing protocol, which is flexible for MobiCCN to switch to the greedy protocol during producer mobility and normal protocol when stop moving [35]. However, even though the proposed scheme maintained one Interest packets to represent both greedy and standard packet, name prefix was reserved to identify the Interest which can result to extra prefix matching time and overhead cost. In addition, when a content producer moves to new PoA, it sends an update packet through intermediate nodes to the home router or rendezvous point for FIB update to forward the Interest towards the new PoA, which is not necessary for greedy Interest to reach the rendezvous, however, this advantage is highly depend on topology not guaranteed.

Joao et al. [36] proposed Controller-based Routing Scheme for NDN to solve the scalability problems caused by content mobility that intensifies high number of content in different locations. The scheme splits content identity and content location from name prefix to facilitate content mobility. The scheme validates and ensures the valid path establishment from consumer to producer, also, performs better for data delivery efficiency [36]. However, the scheme does not ensure path optimization after handoff and when producer mobility rate increases; the overhead will increase and degrade the efficiency.

\section{REVIEW ANALYSIS AND DISCUSSION}

The review analysis is presented based on number of researches conducted according to four different approaches. Figure 5 shows the year, for which many schemes were proposed, the years were plot against the percentage of research conducted according to the literature. In the other graph, the chart shows the analysis of approaches against the percentage level of research conducted.

From the trend of research conducted based on the year since the initial effort for solving producer mobility in NDN, from 2012 to date. Figure 3 (a) indicates the trend of producer mobility support scheme with respect to year. The highest record was in 2017 due to tye fact that many researchers focus on how to make NDN a promising future Internet [8] by solving the inheren problems of IP and currently the Cisco proposed to integrate hybrid ICN the comprises NDN and CCN in to 5G network [37]. This effort made by Cisco encourage many researchres like [38], [39] to explore the relevance of ICN with 5G. The research was also high may be as a result of NDN technical reports on the new perspective of mobility support in NDN [3], that pave a way for the researchers to explore more in providing mobility support to NDBN. Currently in 2017 many research are on the way, this concluded that producer mobility support is a hot area of research in NDN. From the approach review analysis, Figure 3 (b) shows the practice trend of mobility schemes based on approach. IBMA is the most adopted and explored research trend with around $40 \%$ number of proposed schemes, followed by MBMA and CDPMA. IBMA has the higher percentage as a result concept adopted from the solution of mobile IP problems, which is very easier and eccficient to apply in NDN. Moerover, the NDN architecture was designed to become the infrastructreless future Interenet architecture. The MBMA approach provides optimal data path for packets delivery after handoff, but employes DNS-like servers as additional infrastructure, which can make the complication for the management of large NDN network. The CDPBMA is not in line with NDN architecture, rather is purely software design networking paradigm. Probably CDPBMA was explored more due the implementation SDN as efficient solution of current Internet architecture with regards to content distribution. 


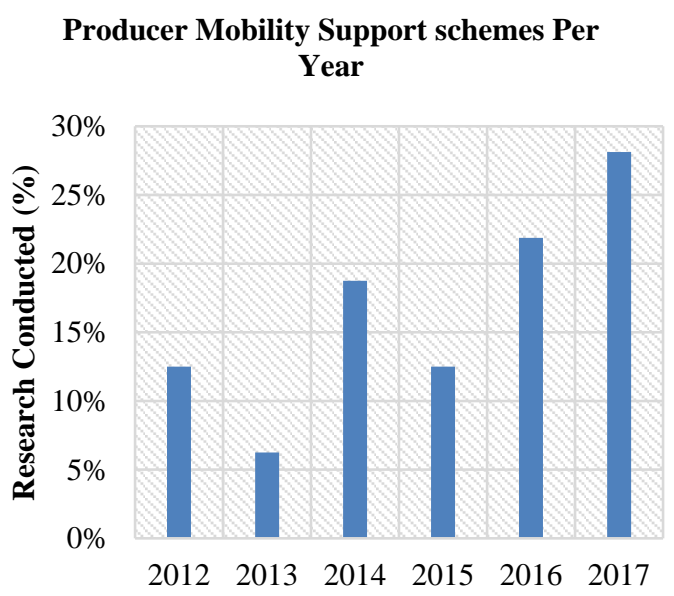

(a)

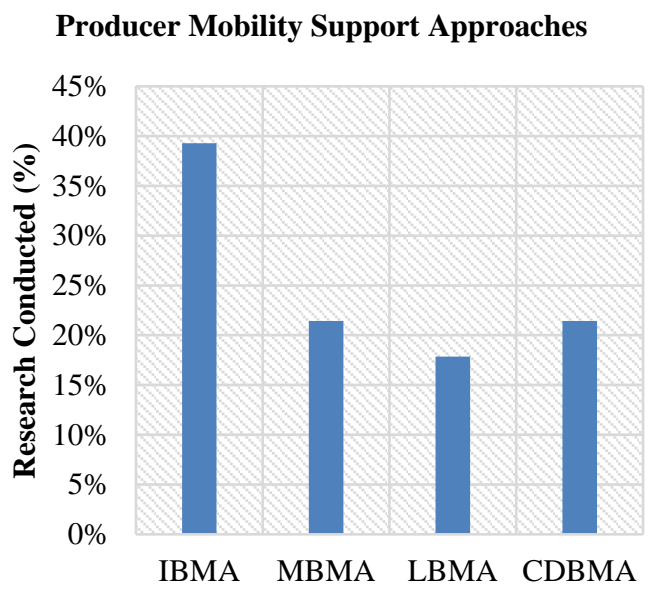

(b)

Figure 3. Yearly Research Conducted on Producer Mobility Support Approaches

\section{CONCLUSION}

The existing solutions of producer mobility support for NDN was reviewed intensively in the literature, based on their approach such as mapping-based, indirection-based, locator-based and control/data plane-based approach. In the review analysis of this paper, mapping-based approach schemes provides optimal path for packets delivery, but with high handoff delay due to the high signalling cost for server mapping processes. Indirection-based and locator-based approach schemes provide normal handoff delay, but introducing tunneling processes, tiangular routing path and the packets transmission through home router may result to a single point of failure. The control/data plane-based approach schemes provides low packets delivery cost, sub-optimal and triangular routing path, high handoff latency, high signalling cost and delay. In general, Indirection-based approach explored more by the researchers and the year 2017 pave the way for many researchrs to explore more about producer mobility support, as a result plan to integrate NDN in to 5G network by Cisco. The survey paper provide some strength and weeakness for each scheme to help in the direction of future work for NDN producer mobility support solution.

\section{REFERENCES}

[1] L. Zhang et al., "Named Data Networking," ACM SIGCOMM Comput. Commun. Rev., vol. 44, no. 3, pp. 66-73, 2014.

[2] B. Feng, H. Zhou, and Q. Xu, "Mobility support in Named Data Networking: a survey," Eurasip J. Wirel. Commun. Netw., vol. 2016, no. 1, 2016.

[3] Z. Zhu, A. Afanasyev, and L. Zhang, "A new perspective on mobility support," Named-Data Networking Project, Technical Report NDN-0013, pp. 1-6, 2013.

[4] I. Angri, M. Mahfoudi, A. Najid, and M. El Bekkali, "Exponential MLWDF (EXP-MLWDF) Downlink Scheduling Algorithm Evaluated in LTE for High Mobility and Dense Area Scenario,” Int. J. Electr. Comput. Eng (IJECE)., vol. 8, no. 3, pp. 1618-1628, 2018.

[5] G. Tyson, N. Sastry, R. Cuevas, I. Rimac, and A. Mauthe, "A survey of mobility in Information-Centric Networks," Commun. ACM, vol. 56, no. 12, pp. 90-98, 2013.

[6] B. Ahlgren, C. Dannewitz, C. Imbrenda, D. Kutscher, and B. Ohlman, "A Survey of Information-Centric Networking," IEEE Commun. Mag., vol. 50, no. 7, pp. 26-36, 2012.

[7] S. Sharma, D. Jindal, and R. Agarwal, "Analysing Mobile Random Early Detection for Congestion Control in Mobile Ad-hoc Network," Int. J. Electr. Comput. Eng., vol. 8, no. 3, pp. 1305-1314, 2018.

[8] D. Saxena, V. Raychoudhury, N. Suri, C. Becker, and J. Cao, "Named Data Networking: A Survey," Comput. Sci. Rev. Elsevier, vol. 19, pp. 15--55, 2016.

[9] K. A. Sultanpure, A. Gupta, and L. S. S. Reddy, "An efficient cloud scheduling algorithm for the conservation of energy through broadcasting,” Int. J. Electr. Comput. Eng., vol. 8, no. 1, pp. 179-188, 2018.

[10] M. Hussaini, S. A. Nor, and A. Ahmad, "Producer Mobility Support for Information Centric Networking Approaches : A Review,” Int. J. Appl. Eng. Res., vol. 13, no. 6, pp. 3272-3280, 2018.

[11] M. Hussaini, S. A. Nor, and A. Ahmad, "PMSS : Producer Mobility Support Scheme optimization with RWP Mobility Model in Named Data Networking," Int. J. Commun. Networks Inf. Secur., vol. 10, no. 2, pp. 1-11, 2018. 
[12] F. Hermans, E. Ngai, and P. Gunningberg, "Global Source Mobility in the Content-Centric Networking Architecture," in Proceedings of the 1st ACM workshop on Emerging Name-Oriented Mobile Networking Design Architecture, Algorithms, and Applications - NoM '12, 2012, pp. 13-18.

[13] Y. Rao, H. Luo, D. Gao, H. Zhou, and H. Zhang, "LBMA: A novel Locator Based Mobility support Approach in Named Data Networking," China Commun., vol. 11, no. 4, pp. 111-120, 2014.

[14] Y. Rao, D. Gao, and H. Luo, "NLBA: A novel provider mobility support approach in mobile NDN environment," in 2014 IEEE 11th Consumer Communications and Networking Conference, CCNC 2014, 2014, no. CCNC, pp. $188-193$.

[15] I. Aldmour, T. Al-dala, L. Siregar, and R. Budiarto, "SeamSAR: Seamless, Secure And Robust Handover Model For Mobile IPTV Network Using Enhanced FMIPv6," Int. J. Electr. Comput. Eng., vol. 5, no. 2, pp. 371-378, 2015.

[16] A. Hafizah, M. Aman, A. A. Hashim, H. Adibah, and M. Ramli, "Throughput and Handover Latency Evaluation for Multicast Proxy Mobile IPV6,” Bull. Electr. Eng. Informatics, vol. 6, no. 4, pp. 311-316, 2017.

[17] D.-H. Kim, J. H. Kim, Y. S. Kim, H. S. Yoon, and I. Yeom, "Mobility Support in Content Centric Networks," in Proceedings of the second edition of the ICN workshop on Information-centric networking, ICN-ACM, 2012, pp. 13-18.

[18] D. Kim, J. Kim, Y. Kim, H. Yoon, and I. Yeom, "End-to-end mobility support in Content Centric Networks," Int. J. Commun. Syst., vol. 28, no. 6, pp. 1151-1167, Apr. 2015.

[19] Z. Yan, G. Geng, S. Zeadally, and Y. J. Park, "Distributed All-IP Mobility Management Architecture Supported by the NDN Overlay," IEEE Access, vol. 5, no. c, pp. 243-251, 2017.

[20] X. Jiang, J. Bi, and Y. Wang, "What benefits does NDN have in supporting mobility," Proc. - Int. Symp. Comput. Commun., 2014.

[21] S. Gao and H. Zhang, "Scalable mobility management for content sources in Named Data Networking," 2016 13th IEEE Annu. Consum. Commun. Netw. Conf. CCNC 2016, pp. 79-84, 2016.

[22] X. Jiang, J. Bi, Y. Wang, P. Lin, and Z. Li, “A content provider mobility solution of Named Data Networking," in Proceedings - 2012 20th IEEE International Conference on Network Protocols (ICNP), 2012, pp. 1-2.

[23] T. X. Do and Y. Kim, "Optimal provider mobility in large-scale Named- Data Networking," KSII Trans. Internet Inf. Syst., vol. 9, no. 10, pp. 4054-4071, 2015.

[24] F. Hermans, E. Ngai, and P. Gunningberg, "Mobile sources in an Information-Centric Network with hierarchical names: An indirection approach," in National Computer Networking 2011.

[25] J. Lee, S. Cho, and D. Kim, "Device mobility management in Content-Centric Networking," IEEE Commun. Mag., vol. 50, no. 12 , pp. $28-34,2012$.

[26] D. Han, M. Lee, K. Cho, T. Kwon, and Y. Choi, "Publisher mobility support in content centric networks," Int. Conf. Inf. Netw. 2014, no. February 2014, pp. 214-219, 2014.

[27] D. Han, M. Lee, K. Cho, T. T. Kwon, and Y. Choi, "PMC : Publisher Mobility Support for Mobile Broadcasting in Content Centric Networks," ASIA Futur. Internet 2012 Summer Sch. Kyoto, Japan, August. 2012. pp. 214-219, 2012.

[28] Z. Yan, S. Zeadally, S. Zhang, R. Guo, and Y.-J. Park, "Distributed mobility management in Named Data Networking," Wirel. Commun. Mob. Comput., vol. 16, no. 13, pp. 1773-1783, Sep. 2016.

[29] R. A. Rehman and B.-S. Kim, "LOMCF: Forwarding and Caching in Named Data Networking-based MANETs," IEEE Trans. Veh. Technol., vol. 66, no. 10, pp. 1-1, 2017.

[30] M. Amadeo, C. Campolo, and A. Molinaro, "Forwarding strategies in named data wireless ad hoc networks: Design and evaluation," J. Netw. Comput. Appl., vol. 50, pp. 148-158, 2015.

[31] A. Azgin, R. Ravindran, and G. Wang, "On-demand mobility support with anchor chains in Information Centric Networks," IEEE Int. Conf. Commun., 2017.

[32] Z. Zhou, X. Tan, H. Li, Z. Zhao, and D. Ma, "MobiNDN: A mobility support architecture for NDN," in Proceedings of the 33rd Chinese Control Conference, CCC 2014, 2014, pp. 5515-5520.

[33] J. Tang, H. Zhou, Y. Liu, H. Zhang, and D. Gao, "A source mobility management scheme in Content-Centric Networking," 2014 IEEE 11th Consum. Commun. Netw. Conf. CCNC 2014, no. Ccnc, pp. 176-181, 2014.

[34] F. Ren, Y. Qin, H. Zhou, and Y. Xu, "Mobility management scheme based on Software Defined Controller for Content-Centric Networking," in Proceedings - IEEE INFOCOM, 2016, vol. 2016-Septe, no. 2, pp. 193-198.

[35] L. Wang, O. Waltari, and J. Kangasharju, "MobiCCN: Mobility support with greedy routing in Content-Centric Networks,” GLOBECOM - IEEE Glob. Telecommun. Conf., pp. 2069-2075, 2013.

[36] J. V. Torres, I. D. Alvarenga, R. Boutaba, and O. C. M. B. Duarte, "An autonomous and efficient controller-based routing scheme for networking Named-Data mobility," Comput. Commun., vol. 103, pp. 94-103, 2017.

[37] Cisco, "Mobile Video Delivery with Hybrid ICN IP-integrated ICN solution for 5G," pp. 1-14, 2017.

[38] R. Ravindran, A. Chakraborti, S. O. Amin, A. Azgin, and G. Wang, "5G-ICN: Delivering ICN Services over 5G Using Network Slicing," IEEE Commun. Mag., vol. 55, no. 5, pp. 101-107, 2017.

[39] M. Chowdhury, A. Gawande, and L. Wang, "Secure Information Sharing among Autonomous Vehicles in NDN," Proc. Second Int. Conf. Internet-of-Things Des. Implement. - IoTDI '17, pp. 15-25, 2017. 